\title{
Evaluation of Mixed Suspension Mixed Product Removal Crystallization Processes Coupled with a Continuous Filtration System
}

\author{
David Acevedo ${ }^{1}$, Ramon Peña ${ }^{1}$, Yang Yang ${ }^{1}$, Alastair Barton ${ }^{2}$, Paul Firth ${ }^{2}$, Zoltan K. Nagy ${ }^{1 *}$ \\ ${ }^{1}$ School of Chemical Engineering, Purdue University, West Lafayette, IN, 47907, USA \\ ${ }^{2}$ Alconbury Weston Ltd (AWL), Stoke-on-Trent, Staffordshire, ST4 2TE, United Kingdom \\ *Corresponding author: email: znagy @ purdue.edu
}

\begin{abstract}
As the pharmaceutical industry evolves and goes through the paradigm shift from batch to continuous manufacturing, innovative processes need to be developed to replace unit operations that have historically been batch operations. This requires innovation in the continuous crystallization field of study as well as innovation in downstream processes (e.g. filtration, drying, milling, and granulation). Herein a novel and commercially available continuous filter carousel (CFC) system was assessed for its feasibility of continuous filtration while coupled with a continuous mixed suspension mixed product removal (MSMPR) crystallizer. The filtration system was assessed using two different crystallization systems (i.e. cooling and antisolvent) with significantly different kinetics and morphologies to assess the robustness of the integrated platform. With proper optimization of the various filtration parameters for the different crystallization systems a controlled state of operation was achieved in each case. The crystal product from the CFC system shows good consistency with the crystals in the slurry in the MSMPR. Moisture content and productivity of the filtration system were reported and show dependency on crystal properties. The CFC system was equipped with solvent vessels that aided the continuous filtration by acting as a wash or a clean-in-place solvent, preventing or removing filter clogging, respectively.
\end{abstract}


Keywords: continuous filtration, continuous crystallization, MSMPR 


\section{Introduction}

Crystallization is a critical step in the pharmaceutical industry being a key separation and purification process. Furthermore, crystallization accounts for over $90 \%$ of all active pharmaceutical ingredient purification processes. Various works have been performed in the design, optimization and control of crystallization processes in order to improve and maintain crystal product qualities such as crystal size, shape and purity, among others [1]-[7]. However, batch operations are well known to affect the overall efficiency of pharmaceutical manufacturing processes and often affect the quality of products due to the significant batch-to-batch variability. Therefore, the study on the design and control of continuous operations has increased significantly. Continuous processing offers the advantage of consistency in product quality and achievement of operating conditions unattainable in batch processes. Other advantages of implementing continuous processing involve the reduction of cost by asset utilization, shorter down time and ease of scale up. Continuous processing has been identified as a key paradigm shift in the pharmaceutical industries with high potential of improving pharmaceutical production [8]-[10].

The two main type of continuous crystallizer designs for pharmaceutical applications are the plug flow and mixed suspension mixed product removal (MSMPR) crystallization systems. Advanced optimization and control strategies have been implemented in these types of crystallizers such as recycle and fines reduction [11]-[14]. The choice of which type of continuous processing to use depends mainly on the kinetics of the process. However, the MSMPR system offers the advantage of simple transfer of existing batch capacity to continuous [8]. The MSMPR crystallizer is an idealized vessel in which supersaturation is generated continuously while crystals nucleate and grow from a feed of homogeneous solution. It is assumed that the product slurry removed continuously has the same composition and crystal 
properties as the vessel content. This is an important assumed property of an MSMPR crystallizer and leads to specific designs of downstream processes.

Filtration has long been developed through practical and empirical understanding instead of exact theory because of the complex nature of the development of the filter cake [15],[16]. Due to this, many issues have arisen in terms of scaling from laboratory to industrial scale and process transfer from batch to continuous operation. Traditionally, laboratory filtration has taken place in batch mode with vacuum as the main driving force. At the manufacturing scale, filtration has mostly been carried out in batch mode consisting of very different geometries and introducing greater uncertainties because of interactions between filter feed and mechanical movement of the filter. Filtration is often assessed in terms of two parameters: mass of solids recovered from the slurry and the moisture content of the recovered solids [16]. Since the majority of the liquid content in a solid-liquid suspension is removed during the filtration process, the effectiveness of unit operations that proceed filtration are directly correlated to these two factors. Drying, for example, is a unit operation that typically proceeds filtration, but is actually only accountable for a small fraction of the liquid removed from the solids. Therefore, incremental improvements in the filtration process can significantly improve the efficiency and energy consumption of a drying unit [17].

Crystallization precedes filtration, and poor filterability of the crystallization product is a common problem for industrial scale pharmaceutical crystallizations. However, it has been shown in the literature that improvements in crystal size distribution (CSD) can greatly improve the efficiency and time of filtration [16],[18],[19]]. The filtration efficiency impacts the crystallization design as different operating conditions produce markedly different crystal properties. Jones et al. [18] conducted a study analyzing the filterability of potassium sulphate 
crystals from a batch crystallization carried out through cooling or anti-solvent addition for both seeded and unseeded cases. They found that the unimodal nature of unseeded crystallization processes greatly improved the permeability of the filter cake allowing for greater liquid removal and lower moisture content. The bimodal nature of seeded crystallization processes introduced greater uncertainty in the filter cake development causing reduced permeability. The study showed no significant effect on filterability from changes in the cooling rate. However, for seeded processes Matthews et al. [19] proposed an optimization framework to reduce the mass of nucleated crystals by controlling the temperature profile and found significantly reduced filtration times when fines were reduced. Jones et al. [18] also pointed out that there is a greater room for improvement in filterability for anti-solvent crystallization as opposed to cooling due to the smaller crystal size, faster rate of supersaturation generation, and potential for agglomeration of the crystals. Fines reduction is not only important during crystallization but during and after filtration, too. Nucleation at the point of filtration is also commonly observed in industrial processes caused by rapid removal of saturated solution from the filter and residual solution dried up on filter parts [20],[21].

There have been numerous studies on the integration of continuous filtration systems coupled to continuous crystallization systems within integrated continuous manufacturing (ICM) systems [22], [23]. However, this studies do not delve into much detail regarding the actual filtration process efficiency, productivity and operating parameters. This is partly due to simple filtration setups with minimal operating parameters built around other unit operations. A few continuous filtration setups are worth mentioning, in Ley et al. [24] work two systems are discussed: (1) the rotating sintered glass filter system which is usually used for liquid collection, is a simple setup with a rotating filtration plate that collects solids while the filtrate can be sent to other unit 
operations; (2) vacuum assisted filter system that works similar to the sintered glass filter system but is used for solids collection equipped with a slurry dispenser, scraper, wash solvent. The vacuum assisted system was used by Mascia et al. [9]. Lastly, Gursch et al. [25],[26] have conducted studies of a dynamic cross-flow filtration (CFF) system which is a membrane based system for the filtration of model active pharmaceutical ingredients (APIs). Their results show the CFF to be a suitable continuous filtration technology with a material-specific linear relationship between feed and permeate rate. However, the effects of shear on the crystals was not studied although membrane based systems have a high dependence on shear rate.

In this work, the feasibility of coupling a continuous filtration carousel (CFC) system with a continuous crystallization process is demonstrated. The aim is to demonstrate the impact of the type of crystallization: (a) cooling and (b) antisolvent on the operation, productivity and product obtained from the continuous filtration system. Paracetamol (PCM) in ethanol is used as the model system for the case study for the cooling crystallization due to the high nucleation and slow growth kinetics, which make the product crystals difficult to filter. Furthermore, benzoic acid (BA) in ethanol drowned out by water was used as the model system for the antisolvent crystallization case study. This system produces crystal morphologies (i.e. needles and plates) common to many active pharmaceutical ingredients (APIs). The variation in the crystal properties produced from the aforementioned crystallization processes assessed the practicability of the continuous filtration system coupled with a MSMPR crystallizer for pharmaceutical crystallization processes.

\section{Materials and Methods}

\subsection{Materials}


4-Acetaminophenol (paracetamol, PCM, Alfa Aesar) with a purity of $98.0 \%$ in mass fraction was used in the cooling crystallization experiments. Benzoic acid (BA, Fisher Scientific Education) with a purity of $99.0 \%$ was used during the antisolvent crystallization case studies. Solubility data for paracetamol in ethanol and benzoic acid in water-ethanol were obtained from the literature [27],[28].

\subsection{System setup}

The continuous MSMPR crystallizer consists of a $500 \mathrm{~mL}$ round-bottom jacketed vessel and a transfer line, as shown in Figure 1a. The transfer line has a similar structure and working mechanism as the one described in literature [29]-[33]. The transfer line contains a transfer zone and four valves, which are used to control nitrogen gas, vacuum, inlet and outlet of the transfer zone, respectively. The slurry is transferred intermittently. Within one transfer, first the vacuum valve is opened for 5 seconds to evacuate the transfer zone, followed by 5 seconds opening of the inlet valve that allows slurry to be vacuumed into the transfer zone. Then the nitrogen valve as well as the outlet valve are opened simultaneously for 5 seconds to push the slurry out into the next vessel. The volume of the transfer zone is $32 \mathrm{~mL}$. The transfer interval is controlled by a controller box to maintain constant slurry levels in both vessels. 
(a)

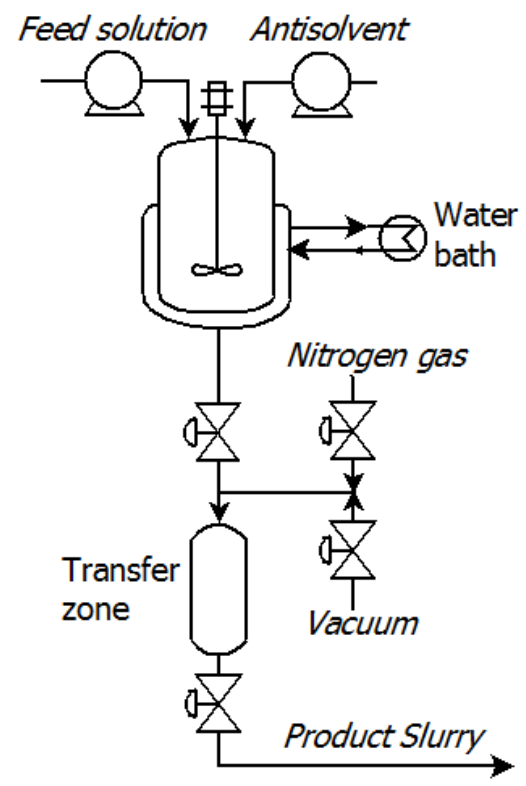

(b) Slurry from continuous crystallizer

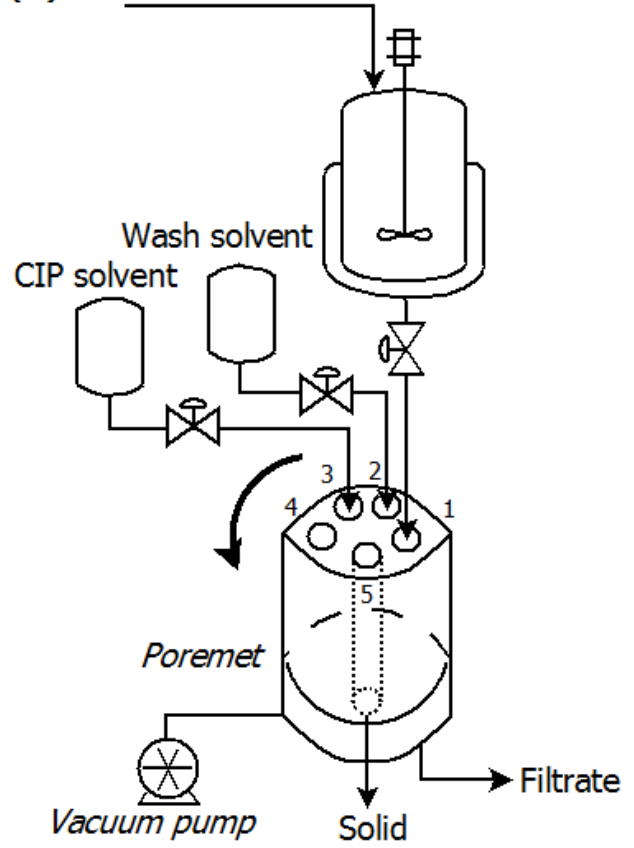

Figure 1. Schematics of (a) continuous MSMPR crystallizer using a transfer line and (b) CFC system.

A commercially available CFC system (Alconbury Weston Ltd, UK) is connected with the continuous MSMPR crystallizer to filter the product slurry. As presented in Figure 1b, the CFC system contains a 2.5-liter slurry vessel, a wash solvent vessel, a clean-in-place (CIP) solvent 
vessel, a filtration unit, and three valves that are used to control slurry, wash solvent and CIP solvent, respectively. The filtration unit consists of five cylindrical-shaped chambers (0.6" diameter and 3.5" height) and a poremet 10 (five layers plate-type filter medium, 2.5 " diameter, 21 microns geometrical pore size, $1.7 \mathrm{~mm}$ thickness) at the bottom. The filter medium spans the chambers located in positions $1,2,3$ and 4 . The chambers rotate counterclockwise every one filtration time. Slurry is fed into the chamber at position 1, as shown in Figure 1b. The slurry in that chamber is then washed in position 2 before being filtered through positions 3 and 4, and finally discharged at position 5 . The bottom of position 5 is open, which allows the cake to be discharged through a chute by a piston. Therefore the filtration unit actually works as a sequential-batch or semi-continuous operation, similar to a semi-batch Nutsche type filter. The bottom of the filtration unit is connected with a vacuum pump, which typically works at around $200 \mathrm{mmHg}$. The filtration time, as well as the slurry valve open time (or slurry time) and the wash solvent valve open time (or wash time) within every one filtration time are parameters that are controllable from the built-in user interface. Each chamber, therefore, operates under variable pressure and filtration rate as these parameters can be controlled and adjusted by the filtration, slurry, or wash timer. In addition, the inner pressure is monitored by the CFC system. Once the pressure drop increases past a user-defined normal operation pressure level, due to blockage of the poremet, an auto-clean function is actuated automatically by feeding CIP solvent into the chambers to dissolve the solids that cause blockage. The filtration unit returns to normal operation once the pressure drop normalizes after auto-clean. However, the small cross-sectional area of each chamber allows for slow ramp in pressure drop which allows for continued productivity without blockage. The appearance of the coupled continuous MSMPR crystallizer and CFC is shown in Figure 2. 


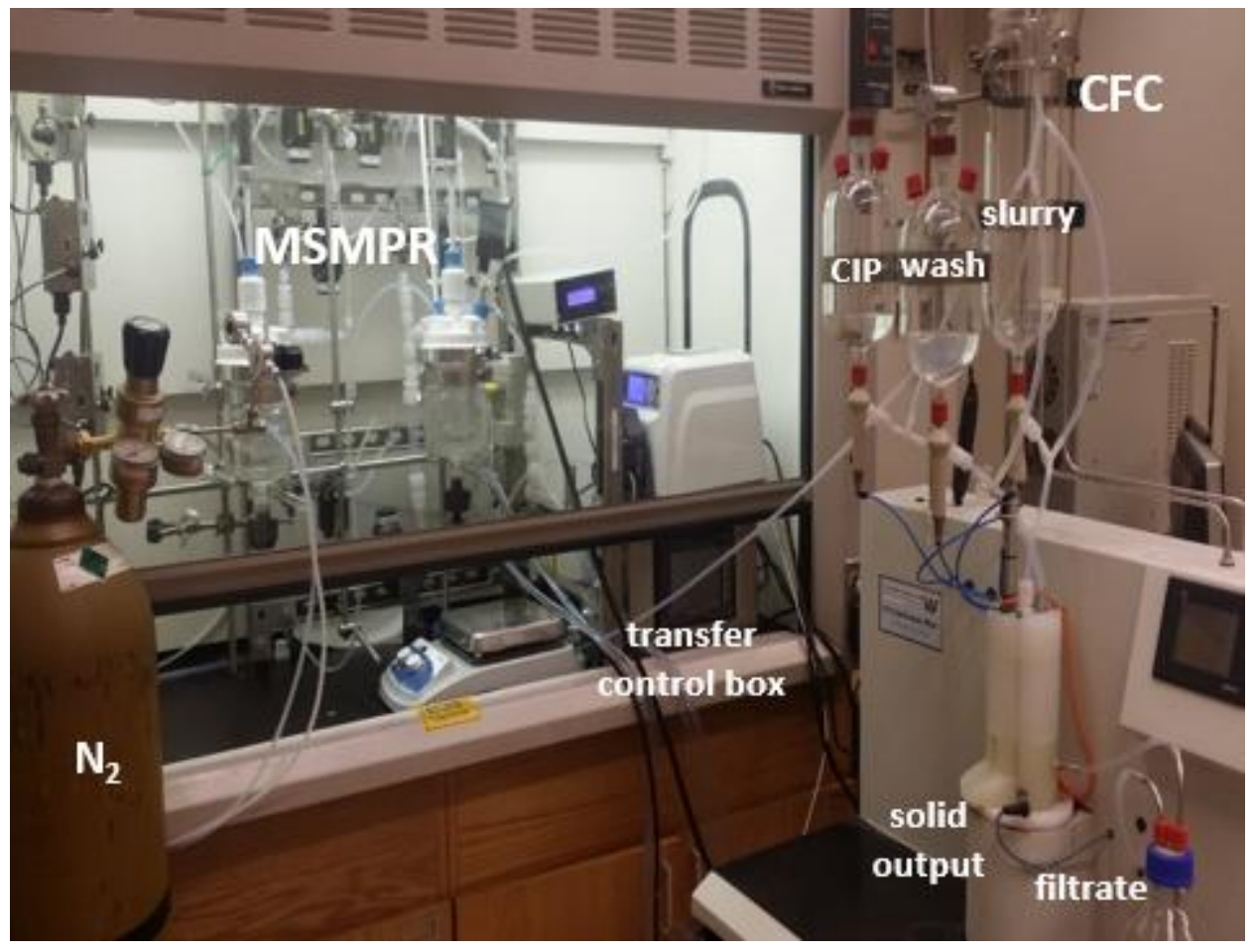

Figure 2. Coupled continuous MSMPR crystallizer and CFC.

\subsection{Methods}

The continuous crystallization was performed in the MSMPR-CFC system described in section 2.2. The temperature in a $500 \mathrm{~mL}$ lab scale glass jacketed vessel was controlled with a PT100 thermocouple using a Huber Ministat 125 refrigerated and heating circulator. An overhead stirrer with a three blade retreat curve impeller was used to agitate the system. The saturated solution or antisolvent was fed to the MSMPR system using a Masterflex L/S pump. An S400 focused beam reflectance measurement (FBRM) probe was used to monitor the dynamics and chord length distribution in-situ during the continuous experiments in order to infer the product CSD [34],[35]. The data was collected every $15 \mathrm{~s}$ in the range of $0.1-1000 \mu \mathrm{m}$. The crystals obtained from the CFC were weighed every half residence time in order to monitor the productivity and dynamics of the filtration system. Samples of filtrated crystals were collected 
every residence time to measure the moisture content by weight loss. Two types of continuous crystallization experiments were performed using paracetamol (PCM) and benzoic acid (BA).

The cooling crystallization of PCM in ethanol was performed in a one stage MSMPR crystallizer as described in section 2.2. A saturated feed solution was prepared by dissolving PCM in $2 \mathrm{~L}$ of ethanol in a beaker at a concentration of $0.24 \mathrm{~g} / \mathrm{g}$ solvent. The initial saturated solution in the crystallizer was prepared at a similar concentration as the feed solution. The feed temperature was set to above the saturation temperature in order to avoid crystallization throughout the feed tubes. The crystallizer temperature was set to $20{ }^{\circ} \mathrm{C}$ to avoid significant impact of temperature variation between the MSMPR stage and CFC slurry stage shown in Figure 1. The agitation speed was set to be $300 \mathrm{rpm}$ throughout the experiments, which is enough to guarantee the suspension of particles. The total residence time was set to 60 minutes to ensure significant growth of the crystals. This is the maximum total residence time allow by using the experimental setup described in section 2.2. The set of experimental conditions for the MSMPRCFC system for the cooling crystallization experiments are shown in Table 1 . The crystals were continuously washed with solvent to minimize the CIP steps which can affect the steady state operation.

Table 1. Experimental conditions for cooling crystallization of PCM in ethanol in a 1 stage MSMPR crystallizer coupled with continuous filtration (CFC) system.

\begin{tabular}{cccccccc}
\hline Variable & Description & Value & Units & Variable & Description & Value & Units \\
\hline$C_{0}$ & initial conc. & 0.24 & $\mathrm{~g} / \mathrm{g}$ & $F$ & flow rate & 5.0 & $\mathrm{~mL} / \mathrm{min}$ \\
$\mathrm{RPM}$ & agitation & 300 & $\mathrm{rpm}$ & $\tau$ & res. time & 60 & $\mathrm{~min}$ \\
$T_{F}$ & feed temp. & 50 & ${ }^{\circ} \mathrm{C}$ & $\tau_{F}$ & filter time & 90 & $\mathrm{~s}$ \\
$T_{C}$ & cryst. temp. & 20 & ${ }^{\circ} \mathrm{C}$ & $\tau_{S}$ & slurry time & 0.4 & $\mathrm{~s}$ \\
$T_{\text {sat }}$ & sat. temp. & 40 & ${ }^{\circ} \mathrm{C}$ & $\tau_{W}$ & wash time & 0.4 & $\mathrm{~s}$ \\
\hline
\end{tabular}


The antisolvent crystallization of BA in ethanol-water was performed in a one stage MSMPR crystallizer as described in section 2.2. The feed solution was prepared by dissolving BA in 700 $\mathrm{mL}$ of ethanol in a beaker at a concentration of $0.285 \mathrm{~g} / \mathrm{g}$ solvent at $50{ }^{\circ} \mathrm{C}$. The initial solution in the crystallizer was an ethanol-water mixture prepared at the same solution to antisolvent ratio (SASR) of the inlet streams. The SASR used for this experiment was 0.43 determined by the ratio of the solution flow rate $\left(S_{\mathrm{FR}}\right)$ to antisolvent flow rate $\left(A S_{\mathrm{FR}}\right)$. This allowed for a slow progression in the crystallization of BA crystals as opposed to initializing with pure water which causes a crashing out effect. The inlet streams, crystallizer and CFC unit were all operated at ambient temperature $\left(\sim 20^{\circ} \mathrm{C}\right)$. The agitation speed was set to be $350 \mathrm{rpm}$ throughout the experiments, which is enough to guarantee the suspension of particles. The total residence time was set to 40 minutes, which was enough to show significant growth of the crystals. The set of experimental conditions for the MSMPR-CFC system for the antisolvent crystallization experiments are shown in Table 2. During operation two CIP procedures were needed due to pressure drop increases from clogging of the filter plate.

Table 2. Experimental conditions for antisolvent crystallization of BA in ethanol-water in a 1 stage MSMPR crystallizer coupled with continuous filtration (CFC) system.

\begin{tabular}{cccccccc}
\hline Variable & Description & Value & Units & Variable & Description & Value & Units \\
\hline$C_{0}$ & initial conc. & 0.285 & $\mathrm{~g} / \mathrm{g}$ & $F$ & overall flow rate & 10.0 & $\mathrm{~mL} / \mathrm{min}$ \\
$\mathrm{RPM}$ & agitation & 350 & $\mathrm{rpm}$ & $\tau$ & res. time & 40 & $\mathrm{~min}$ \\
$S o l_{F R}$ & sol. flow rate & 3 & $\mathrm{~mL} / \mathrm{min}$ & $\tau_{F}$ & filter time & 120 & $\mathrm{~s}$ \\
$A S_{F R}$ & antisol. flow rate & 7 & $\mathrm{~mL} / \mathrm{min}$ & $\tau_{S}$ & slurry time & 1 & $\mathrm{~s}$ \\
$S A S R$ & Ratio & 0.43 & - & $\tau_{W}$ & wash time & 0.15 & $\mathrm{~s}$ \\
\hline
\end{tabular}




\section{Results and Discussion}

\subsection{Cooling crystallization of paracetamol}

The cooling crystallization of paracetamol was performed in a one stage MSMPR system and monitored using the FBRM. The square weighted mean chord length is shown in Figure 3a. First order dynamics are observed, which is expected since the continuous process was initiated with a saturated solution in the MSMPR vessel. The dynamic variation of the square weighted mean chord length demonstrates that the system achieved steady state behavior around 3 residence times $(180 \mathrm{~min})$. The oscillations in the dynamic profile, shown in Figure 3a, are commonly observed during the continuous crystallization in a MSMPR system [12],[36]-[38]. The oscillations in the square weighted mean chord length are within $\pm 8 \mu \mathrm{m}$. Therefore, the system is considered to reach steady state behavior (controlled state of operation, CSO) after three residence times. The square weighted mean chord length achieved at steady state shows that the system does not grow significantly, which is expected for a nucleation dominated system, such as PCM [2]. The small size achieved will impact the filtration behavior as observed in Figure 3a.
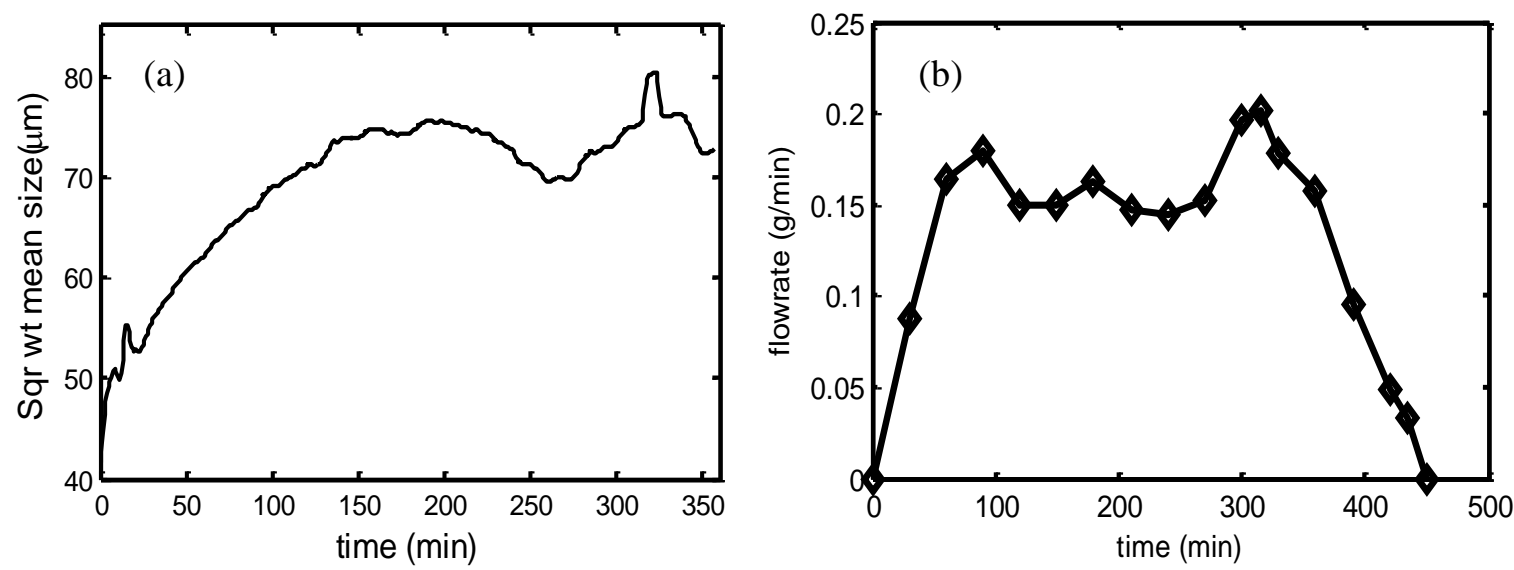

Figure 3. (a) Square weighted mean chord length $(\mu \mathrm{m})$ and (b) mass flowrate from CFC (g/min) during cooling crystallization of PCM in a MSMPR crystallizer coupled with CFC system. 
The mass flowrate of PCM crystals obtained from the continuous filtration system (CFC) shows higher order dynamics. The continuous filtration processes was not started until half residence time in order to allow the filtration vessel to reach a minimum working volume in which the system could be operated with low fluctuations. Furthermore, the system was operated at low volume to avoid further growth or dissolution of crystals. Figure 4 shows microscope images of slurry samples taken after the system reached steady state, and filtered crystals after drying. No significant growth was observed between samples from the MSMPRS and filtered crystals. There is no clear evidence of breakage or attrition on the final crystals, which could affect the final crystal quality. Figure 4 demonstrates no significant variation in the crystal habit of PCM before and after the continuous filtration. Therefore, there is no observable impact due to growth, breakage or attrition on the PCM crystals that can occur while the slurry is in the filtration feed vessel or in the filtration chambers. These results indicate that the CFC system is a good isolation platform for the studied system as the crystallization product properties are preserved during the filtration.
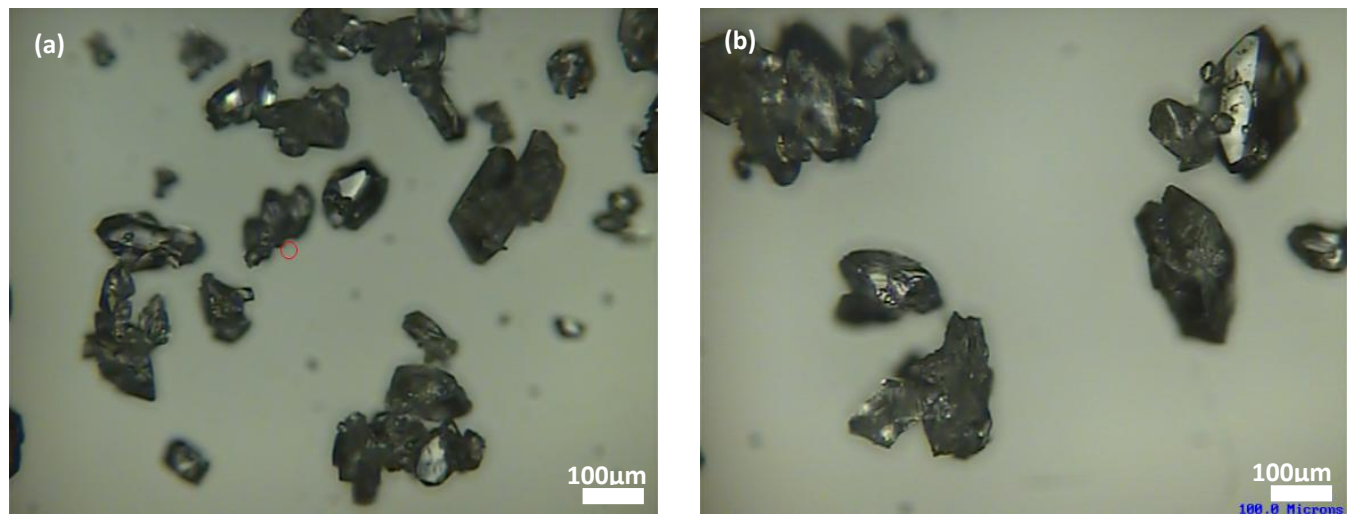
Figure 4. Microscope images obtained of (a) slurry at outlet of MSMPR and before the CFC and (b) filtered crystals after the CFC. Samples were obtained at steady-state.

The startup of the filtration system depends on the control of the volume at which the CFC is operated but also on the dynamics of the continuous crystallization process. As observed, steady state behavior was achieved between 2 to 2.5 hours of operation. A mean mass flow rate of $0.17 \pm 0.03 \mathrm{~g} / \mathrm{min}$ during the steady state operation was achieved. The low productivity obtained can be attributed to the use of wash during the process in order to avoid disturbances in the continuous operation due to the CIP programmed step. However, higher productivity can be achieved by increasing the filtration timer which will maintain the same throughput and loose some running time to CIP. The continuous crystallization experiment was continued for 3.5 residence times after the system reached steady state as observed in Figure 3a. However, the continuous filtration system was operated until all the slurry in the vessel was filtered. This allowed the observation of the impact of decreasing working volume on the mass flowrate of crystals as demonstrated in Figure 3b. A monotonic decrease in the mass flowrate was observed after stopping the transfer from the MSMPR vessel to the filtration system. The startup and shutdown period observed in Figure $3 \mathrm{~b}$ demonstrates that it is necessary to operate the $\mathrm{CFC}$ at constant volume since this has a direct impact in the output from the system; the amount of slurry pushed from the vessel when the filtration valve opens depend on the working volume dynamics (refer to section 2.2).

The moisture content was determined for the samples collected at each residence time from the filtered crystals at the outlet of the CFC. Figure 5 shows the moisture content (MC) dynamics 
calculated by weight loss after a $24 \mathrm{~h}$ drying period. The dynamics demonstrated that the system reaches a steady state behavior after two residence times. An average MC of $22.2 \pm 2.8 \%$ was achieved at steady state. The MC before reaching steady state is significantly lower due to the lower amount of material in the filtration chambers. As demonstrated before, the mass flowrate before reaching steady state is lower, thus the vacuum system can remove solvent more efficiently. If the filtration operating conditions are constant throughout the processes, higher removal of solvent should be expected during startup due to the lower amount of material in the chambers.

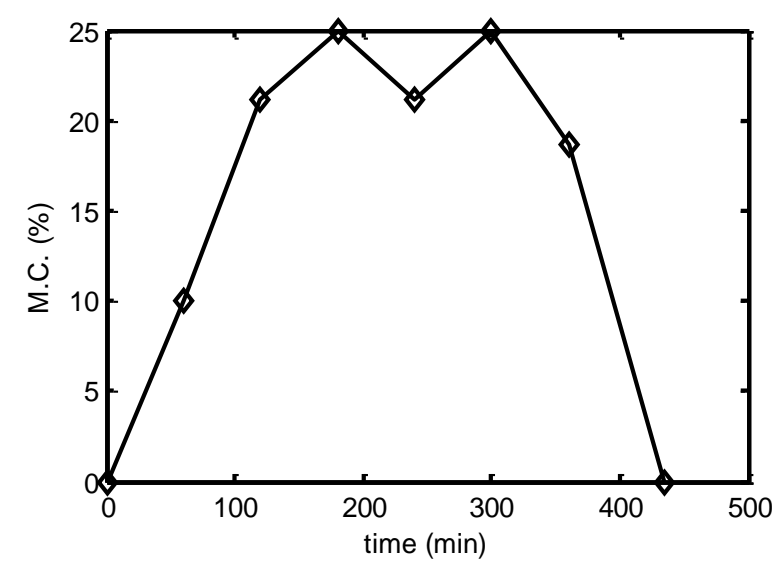

Figure 5. Moisture content (\%) of filtered crystals obtained at each residence time throughout the cooling crystallization of PCM in a one stage MSMPR crystallizer coupled with the CFC system.

\subsection{Antisolvent crystallization of benzoic acid}

The antisolvent crystallization of benzoic acid was performed in a one stage MSMPR system and monitored using the FBRM. The square weighted mean chord length is shown in Figure 6a. Higher order dynamics is observed, during the startup indicated by the initial overshoot and oscillations in the system behavior, which is generally expected for a continuous antisolvent crystallization process. The dynamic variation of the square weighted mean chord length demonstrates that the system achieved a steady state behavior at around four residence times 
(160 min). The oscillations in the dynamic profile after reaching steady state in this case study were negligible and can be attributed to intermittent operation. The steady state mean chord length was around $110 \mu \mathrm{m}$. This mean size showed good filtration properties aside from the two CIP procedures required at 120 and $200 \mathrm{~min}$.
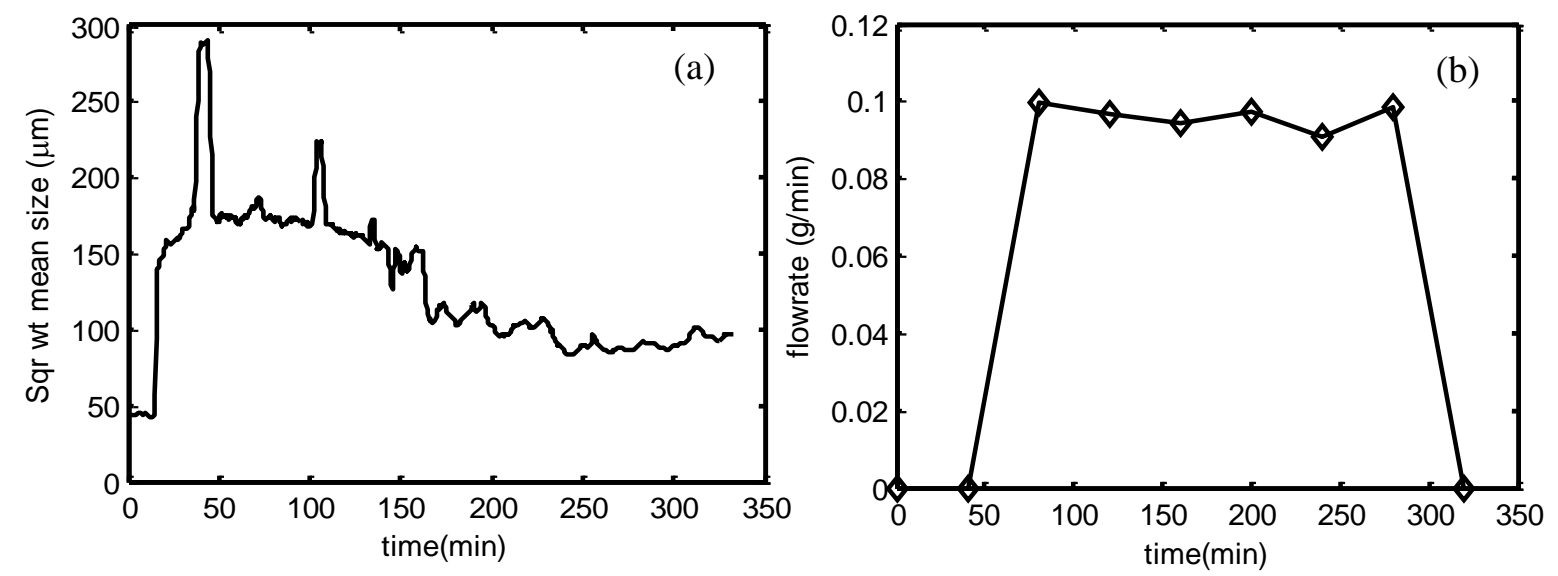

Figure 6. (a) Square weighted mean chord length $(\mu \mathrm{m})$ and (b) mass flowrate $(\mathrm{g} / \mathrm{min})$ during antisolvent crystallization of BA in a MSMPR crystallizer coupled with the CFC system.

The mass flowrate of BA crystals (Figure 6b) obtained from the CFC shows well maintained dynamics and was unaffected by the CIP procedures. Similar to the case of PCM, the continuous filtration process was not started until half residence time and there was no observable impact on BA crystal attributes due to the added holdup filtration period. The startup of the filtration system depends on the operating volume the CFC but also on the dynamics of the continuous crystallization process. Since antisolvent crystallization technique was used for BA there the induction time for crystallization was shorter and there was a significant amount of crystal mass in the slurry from the onset, although the solid concentration was relatively small. A mean mass flow rate of $0.10 \pm 0.003 \mathrm{~g} / \mathrm{min}$ during the steady state operation was achieved. The low productivity obtained can be attributed to low solids concentration in the slurry. Given that 
filtration was not initiated until the appropriate working volume was achieved the mass flow rate for the antisolvent system was sufficiently constant. This is also attributed to the fact that the antisolvent system produces a fairly constant solids concentration from the onset due to the short induction time

Figure 7 shows the morphology of the BA crystals obtained from the antisolvent crystallization. As the microscope images show, the morphologies present are both rigid plates and needles. This had a significant impact on the filtration process, specifically on the moisture content. The moisture content was determined for the samples collected at each residence time from filtered crystals at the outlet of the CFC.
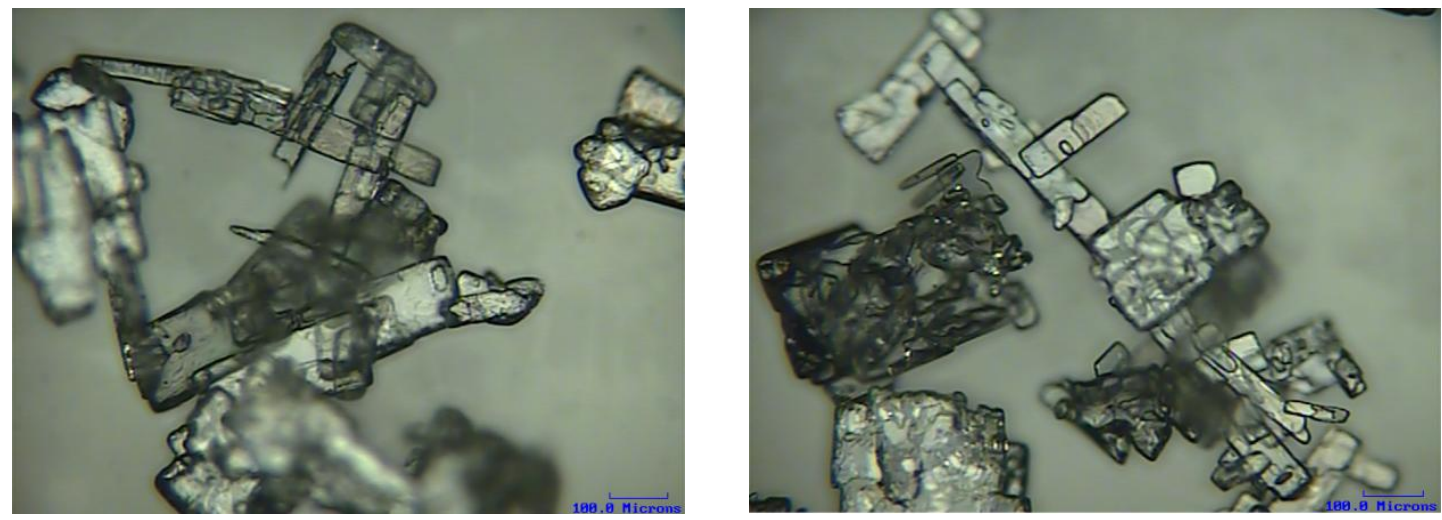

Figure 7: Microscope images of filtered crystal samples obtained at steady-state.

Figure 8 shows the MC dynamics obtained for BA after a 24 hours drying period; an average MC of $45 \pm 4 \%$ was achieved at steady state. The high moisture content can be attributed to the different morphologies of the benzoic acid crystals present. This directly impacts the development of the filter cake inside the filtration chambers and has a liquid entrapment affect. This is evident from the microscope images in which the crystals appear clustered together due the liquid entrapment. From Figure 8 two points of decreased moisture content are observed at 
120 and $200 \mathrm{~min}$. These points coincide with the two CIP procedures. The results show that after a CIP there is a significant improvement in the amount of solvent removed and shortly after the moisture content increases again. This proves that there is a combination of crystals that clog the pores of the filter medium as well as liquid entrapment in the filter cake that cause gradual increase in moisture content. The crystals clogging the pores of the medium are easily removed by the CIP solvent allowing for continued operation with small deviation in the process output.

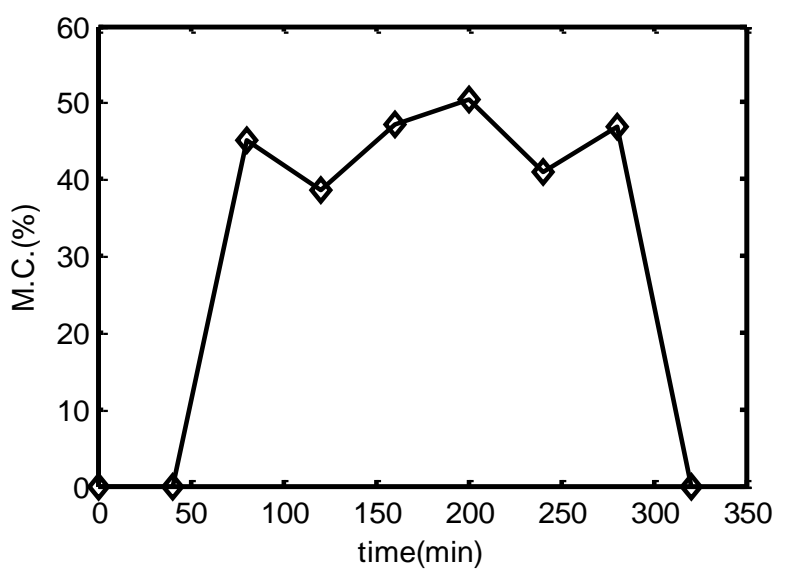

Figure 8. Moisture content (\%) of filtered crystals obtained at each residence time throughout the antisolvent crystallization of BA in a one stage MSMPR crystallizer coupled with the CFC system.

\subsection{Assessment of continuous filtration system}

The CFC system presented in our work also allows washing the filter as a wash vessel and injection port onto the filter cake is available. Cleaning is also made easier by the presented system given the CIP vessel, which can contain a solvent that has a high solubility for the solids being recovered so that efficient cleaning can take place. However, whenever the CIP takes place the filtration step shows small deviation from steady state operation and the properties, including the moisture content, change. The impact of the CIP on the filtration step was observed for both scenarios studied but it was more frequent for the antisolvent crystallization of BA compared to the cooling crystallization of PCM. This could be attributed to the differences in crystal habit 
between the two systems as observed in Figure 7 and Figure 4b. Needle-like and plate shape crystals were obtained for BA during steady state operation. The PCM crystals obtained show a prismatic crystal habit. Hence, clogging occurred more frequently during the crystallization of BA due to the wide crystal shape distribution. The operating conditions of the CFC system would depend on the type of process and crystallization system. An important observation is that the performance of the filtration step depends significantly on the crystallization system and process. The achievable productivity and moisture content are correlated with the filtration parameters, which will also depend on the crystallization system. However, the small variability in the productivity and moisture content observed for both processes, demonstrate the robustness of the continuous crystallization process coupled with the CFC system. The highest variation in the moisture content occurs for both systems whenever a CIP step initiates. Therefore, the variance observed throughout the operation of the CFC should be minimized if optimal operating conditions are implemented. Furthermore, the filtration buffer vessel should be operated at small residence times in order to avoid significant impact on the quality of the crystals produced from the continuous crystallization process. The temperature in the filtration buffer vessel should also be controlled, to avoid additional nucleation or growth. In our study the MSMPR stage was operated close or at ambient temperature in order to avoid any impact of the filtration buffer vessel temperature variation on the product.

The presented study demonstrated that the CFC system is a viable process for the continuous filtration of pharmaceuticals compounds and can be easily coupled with continuous crystallization to form a fully integrated platform. The ease of use and controllability of the CFC system make it feasible to operate continuously, while coupled with a continuous crystallization process 


\section{Conclusions}

The operation of a continuous crystallization coupled with a novel continuous filter carousel (CFC) system was demonstrated through different case studies, (a) cooling crystallization of paracetamol and (b) antisolvent crystallization of benzoic acid. The continuous filtration showed significant dependence on the crystallization system due to the variations in crystal properties such as size and shape. This could lead to operation of the CFC system at sub-optimal conditions that could affect the efficiency of the process. However, the robustness of the system is demonstrated due to the low variability during steady-state operation. The continuous operation of the MSMPR coupled with the CFC system shows significant promise due to the robustness and fast start-up observed for the cooling and antisolvent crystallization of paracetamol and benzoic acid, respectively.

\section{Acknowledgments}

The authors would like to thank Eli Lilly \& Company for supplying the transfer line unit used throughout the various experiments. Also, the support from Alconbury Weston Ltd. by supplying the continuous filtration system is gratefully acknowledged. This material is based upon work supported by the National Science Foundation Graduate Research Fellowship under Grant no. DGE-1333468. Any opinion, findings, and conclusions or recommendations expressed in this material are those of the authors and do not necessarily reflect the views of the National Science Foundation. 


\section{References}

[1] Z.K. Nagy, R.D. Braatz, Advances and new directions in crystallization control, Annu. Rev. Chem. Biomol. Eng. 3 (2012) 55-75.

[2] D. Acevedo, Z.K. Nagy, Systematic classification of unseeded batch crystallization systems for achievable shape and size analysis, J. Cryst. Growth. 394 (2014) 97-105.

[3] D. Acevedo, Z.K. Nagy, Multi-objective optimization of an unseeded batch cooling crystallizer for shape and size manipulation, Ind. Eng. Chem. Res. 54 (2015) 2156-2166.

[4] Y. Yang, Z.K. Nagy, Model-based systematic design and analysis approach for unseeded combined cooling and antisolvent crystallization (CCAC) systems, Cryst. Growth Des. 14 (2014) 687-698.

[5] J.C. King, H. Li, M.A. Grover, Y. Kawajiri, R.W. Rousseau, optimization of two-stage cooling profile in unseeded batch cooling crystallization, IFAC-PapersOnLine 48 (2015) 297-302.

[6] C.B. Costa, R. Maciel, Evaluation of optimisation techniques and control variable formulations for a batch cooling crystallization process, Chem. Eng. Sci. 60 (2005) 53125322.

[7] L.X. Yu, R.A. Lionberger, A.S. Raw, R. D'Costa, H. Wu, A.S. Hussain, Applications of process analytical technology to crystallization processes, Adv. Drug Deliv. Rev. 56 (2004) $349-369$.

[8] J. Chen, B. Sarma, J.M.B. Evans, A.S. Myerson, Pharmaceutical crystallization, Cryst. Growth Des. 11 (2011) 887-895.

[9] S. Mascia, P. L. Heider, H. Zhang, R. Lakerveld, B. Benyahia, P. I. Barton, R. D. Braatz, C. L. Cooney, J. M. B. Evans, T. F. Jamison, K. F. Jensen, A. S. Myerson, B. L. Trout, End-to- 
End Continuous Manufacturing of Pharmaceuticals: Integrated Synthesis, Purification, and Final Dosage Formation. Angew. Chem. Int. Ed. 52 (2013) 12359-12363.

[10] R. Lakerveld, B. Benyahia, P. L. Heider, H. Zhang, A. Wolfe, C. J. Testa, S. Ogden, D. R. Hersey, S. Mascia, J. M. B. Evans, R. D. Braatz, P. I. Barton, The Application of an Automated Control Strategy for an Integrated Continuous Pharmaceutical Pilot Plant. Org. Process Res. Dev. 19 (2015) 1088-1100.

[11] A. Majumder, Z.K. Nagy, Fines removal in a continuous plug flow crystallizer by optimal spatial temperature profiles with controlled dissolution, AIChE J. 59 (2013), 45824594.

[12] D.W. Griffin, D.A. Mellichamp, M.F. Doherty, Reducing the mean size of API crystals by continuous manufacturing with product classification and recycle, Chem. Eng. Sci. 65 (2010) 5770-5780.

[13] R.J.P. Eder, S. Radl, E. Schmitt, S. Innerhofer, M. Maier, H. Gruber-Woelfler, J.G. Khinast, Continuously seeded, continuously operated tubular crystallizer for the production of active pharmaceutical ingredients, Cryst. Growth Des. 10 (2010) 2247-2257.

[14] Y. Yang, Z.K. Nagy, Adanced control approached for combined cooling/antisolvent crystallization in continuous mixed supension mixed product removal cascade crystallizers, Chem. Eng. Sci. 127 (2015) 362-373.

[15] C. Tien, R. Bai, B.V. Ramarao, Analysis of cake growth in cake filtration: Effect of fine particle retention, AIChE J. 43 (1997) 33-44.

[16] T. Reynolds, M. Boychyn, T. Sanderson, M. Bulmer, J. More, M. Hoare, Scale-down of continuous filtration for rapid bioprocess design: recovery and dewatering of protein precipitate suspensions, Biotechnol. Bioeng. 83 (2003) 454-464. 
[17] W. Chen, Optimization of sludge dewatering through pretreatment, equipment selection, and testing, Drying Technol. 31 (2013) 193-201.

[18] A.G. Jones, J. Budz, J.W. Mullin, Batch crystallization and solid-liquid separation of potassium sulphate, Chem. Eng. Sci. 42 (1987) 619-629.

[19] H.B. Matthews, J.B. Rawlings, Batch crystallization of a photochemical: modeling, control, and filtration, AIChE J. 44 (1998) 1119-1127.

[20] N. Zaitseva, J. Atherton, R. Rozsa, L. Carman, I. Smolsky, M. Runkel, R. Ryon, L. James, Design and benefits of continuous filtration in rapid growth of large KDP and DKDP crystals, J. Cryst. Growth 197 (1999) 911-920.

[21] N.E. Chayen, Rigorous filtration for protein crystallization, J. Appl. Cryst. 42 (2009) $743-744$.

[22] H. Zhang, R. Lakerveld, P.L. Heider, M. Tao, M. Su, C.J. Testa, A.N. D’Antonio, P.I. Barton, R.D. Braatz, B.L. Trout, A. S. Myerson, Application of continuous crystallization in an integrated continuous pharmaceutical pilot plant, Crys. Growth Des., 14 (2014), 21482157.

[23] R.J. Ingham, C. Battilocchio, D.E. Fitzpatrick, E. Sliwinski, J.M. Hawkins, S.V. Ley, A Systems Approach towards an Intelligent and Self - Controlling Platform for Integrated Continuous Reaction Sequences. Angew. Chem. Int. Edit., 54 (2015), 144-148.

[24] S.V. Ley, D.E. Fitzpatrick, R. Ingham, R.M. Myers, Organic synthesis: march of the machines. Angew. Chem. Int. Edit., 54 (2015), 3449-3464.

[25] J. Gursch, R. Hohl, G. Toschkoff, D. Dujmovic, J. Brozio, M. Krumme, N. Rasenack, J. Khinast, Continuous Processing of Active Pharmaceutical Ingredients Suspensions via Dynamic Cross-Flow Filtration. J. Pharm. Sci., 104 (2015), 3481-3489. 
[26] J. Gursch, R. Hohl, D. Dujmovic, J. Brozio, M. Krumme, N. Rasenack, J. Khinast, Dynamic cross-flow filtration: enhanced continuous small-scale solid-liquid separation. Drug Dev. Ind. Pharm., 42 (2016), 977-984.

[27] N.A. Mitchell, P.J. Frawley, C.T. Ó'Ciardhá, Nucleation kinetics of paracetamol-ethanol solutions from induction time experiments using Lasentec FBRM, J. Cryst. Growth, 321 (2011) 91-99.

[28] D. O'Grady, Multiscale characterization of antisolvent crystallization, Thesis, National University of Ireland, 2007. U College Dublin, 2007.

[29] Y. Yang, L. Song, T. Gao, Z.K. Nagy, Integrated upstream and downstream application of wet milling with continuous mixed suspension mixed product removal crystallization, Cryst. Growth Des. 15 (2015) 5879-5885.

[30] Y. Yang, L. Song, Z.K. Nagy, Automated direct nucleation control in continuous mixed suspension mixed product removal cooling crystallization, Cryst. Growth Des. 15 (2015) $5839-5848$.

[31] Y. Yang, L. Song, Y. Zhang, Z.K. Nagy, Application of wet milling based automated direct nucleation control in continuous cooling crystallization processes, Ind. Eng. Chem. Res. 55 (2016) 4987-4996.

[32] R. Peña, Z.K. Nagy, Process intensification through continuous spherical crystallization using a two-stage mixed suspension mixed product removal (MSMPR) system, Cryst. Growth Des. 15 (2015) 4225-4236.

[33] G. Hou, G. Power, M. Barrett, B. Glennon, G. Morris, Y. Zhao, Development and characterization of a single stage mixed-suspension, mixed-product-removal crystallization process with a novel transfer unit, Cryst. Growth Des. 14 (2014) 1782-1793. 
[34] H. Li, Y. Kawajiri, M.A. Grover, R.W. Rousseau, Application of an empirical FBRM model to estimate crystal size distributions in batch crystallization, Cryst. Growth Des. 14 (2014) 607-616.

[35] H. Li, M.A. Grover, Y. Kawajiri, R.W. Rousseau, Development of an empirical method relating crystal size distributions and FBRM measurements, Chem. Eng. Sci. 89 (2013) 142151.

[36] Y. Yang, Z.K. Nagy, Combined cooling and antisolvent crystallization in continuous mixed suspension mixed product removal cascade: steady state and startup optimization, Ind. Eng. Chem. Res. 54 (2015) 5673-5682.

[37] Y. Yang, Z.K. Nagy, Application of nonlinear model predictive control in continuous crystallization systems, Proceedings of the American Control Conference (2015) 4282-4287.

DOI: 10.1109/ACC.2015.7172002

[38] B.G. Lakatos, T.J. Sapundzhiev, J. Garside, Stability and dynamics of isothermal CMSMPR crystallizers, Chem. Eng. Sci. 62 (2007) 4348-4364. 


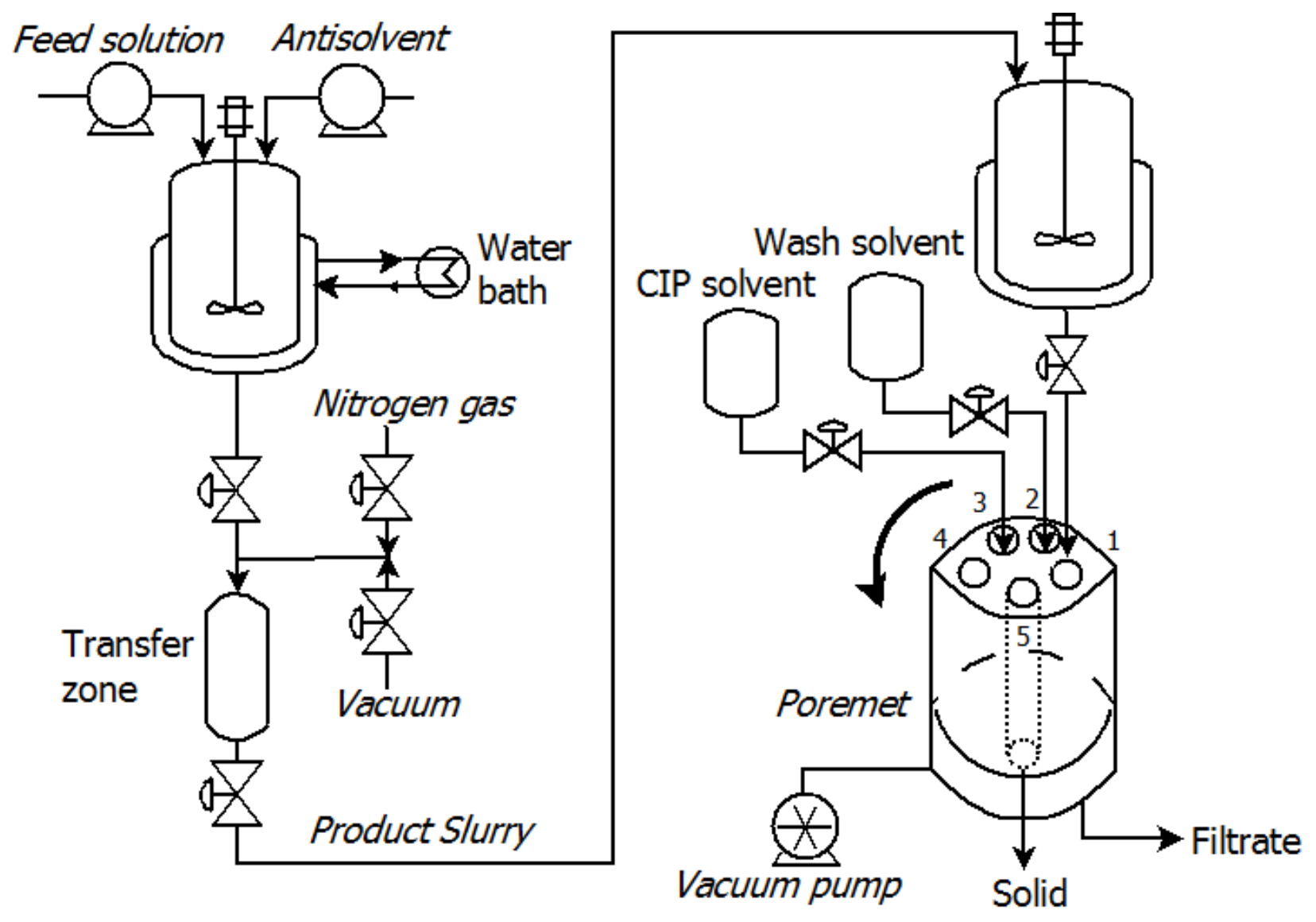

